\title{
VARICOCELECTOMÍA LAPAROSCÓPICA EN EL ADULTO
}

\author{
René Salas Cabrera, Carlos Ramírez Torres, Jorge Sagué Larrea y Ariel Laurencio Mena.
}

Servio de Cirugía Laparoscópica. Sección Uro-Oncológica. Hospital Vladimir Ilch Lenin. Holguín. Cuba.

Resumen.- OBJETIVO: Conocer nuestros resultados con esta novedosa técnica en la Provincia.

MÉTODO: Se realiza un estudio en 120 pacientes con el diagnostico positivo (doppler testicular) de varicocele. Desde junio del año 2004 a julio del 2006, analizando las siguientes variables: Tiempo quirúrgico, estancia hospitalaria, uso de analgésicos post operatorios, complicaciones e incorporación a las actividades sociolaborales.

RESULTADOS: Se intervienen 120 pacientes entre 18 y 42 años de edad. El tiempo quirúrgico promedio fue 25.07 minutos. La minoría de los pacientes egresaron al día siguiente (dificultad con el transporte), el resto se trataron de forma ambulatoria. No se utilizó prácticamente analgésicos post operatorios, sólo 3 pacientes requirieron tratamiento del dolor. Se presentaron sólo 3 complicaciones menores durante o después de la ciru- gía. Todos los pacientes tuvieron una rápida incorporación a las actividades sociolaborales (entre 15 y 21 ).

CONCLUSIONES: Consideramos que la ligadura laparascópica de las venas espermáticas puede considerarse como método eficaz en el tratamiento de pacientes con diagnóstico de varicocele. De elección en el varicocele bilateral, en pacientes obesos, después del fracaso de las técnicas convencionales, con antecedentes de cirugía inguinal y en la necesidad de tratamiento laparoscópico simultáneo de otras patologías. Sin olvidar la importancia que juega como entrenamiento en la curva de aprendizaje para el desarrollo de esta técnica.

Palabras clave: Varicocele. Infertilidad masculina. Varicocelectomía laparoscópica.

Summary.- OBJECTIVES: We performed a study including 120 patients with the diagnosis of varicocele (testicular Doppler ultrasound), from June 2004 to July 2006, analyzing the following variables: surgical time, hospital stay, postoperative analgesic requirements, complications and return to social-working activities.

RESULTS: 120 patients between 18 and 42 years of age underwent surgery. Mean surgical time was 25.07 minutes. A minority of the patients were discharged the following day (difficulties with transportation), the rest were treated as outpatient. Almost no postoperative analgesic drugs were employed, only three patients required pain treatment. Only three minor complications appeared during or after surgery. All patients had a fast recovery back to their social-working activities (between 15 and 21 days).

CONCLUSIONS: We consider laparoscopic ligation of spermatic veins may be considered an effective method for the treatment of patients with varicocele. It is the treatment of choice for bilateral varicocele, obese patients, after the failure of conventional techniques, in 
patients with history of inguinal surgery, and when simultaneous laparoscopic treatment of other pathologies is necessary. We also emphasize the importance it plays as training in the learning curve for the development of this technique.

Keywords: Varicocele. Male infertility. Laparoscopic varicocelectomy.

\section{INTRODUCCIÓN}

El varicocele es una patología conocida desde muy antiguo. Sin embargo sigue rodeado de numerosos enigmas.

Se define como la dilatación varicosa del plexo pampiniforme, resultado del flujo retrógrado venoso anormal hacia los testículos. Se observa en aproximadamente el $15 \%$ de la población general masculina.

La oclusión de la vena espermática interna erróneamente denominada "VARICOLECTOMÍA". Es el tratamiento más idóneo para corregir esta patología.

Se han realizado varios procedimientos para la corrección de esta patología, la mayoría de los urólogos optan por la cirugía convencional. La reparación del varicocele mediante laparoscopia fue descrita por primera vez en 1986 y desde el mismo comienzo ha sido una práctica quirúrgica centro de controversias. No obstante la cirugía laparoscópica ofrece muchas ventajas.

\section{MATERIAL Y MÉTODO}

Se realiza un estudio en 120 pacientes con el diagnóstico positivo (Doppler testicular) de varicocele. Desde junio del año 2004 a julio del 2006.

\section{Modo de proceder}

Previo vaciado vesical espontáneo, el paciente bajo anestesia general y en posición de de- cúbito suspino, con discreto Trendelemburg, puncionamos cavidad peritoneal con aguja de Verres (Pneumoperitoneo), a nivel del contorno inferior umbilical, donde luego de alcanzar una presión de $\mathrm{CO}_{2}$ de $15 \mathrm{~mm} \mathrm{hg}$, introducimos el trocar óptico, Después de inspección visual de la cavidad, realizamos la colocación de trócares accesorios, a la derecha 10 $\mathrm{mm}$ y la izquierda de $5 \mathrm{~mm}$, a nivel pararrectal, a la altura del ombligo. Preferimos colocarnos como cirujanos detrás de la cabeza del paciente, permitiendo el acceso bilateral con mayor comodidad.

Tras identificar los vasos espermáticos, procedemos a efectuar corta incisión del peritoneo parietal, para posteriormente realizar la disección del paquete (arteria y vena) y colocar un solo clip, en algunos casos por presentar cierto grado de dilatación es necesario usar más de un clip para garantizar la ligadura de todos los vasos, revisamos hemostasia y termina la operación con el cierre músculo-aponeurótico y piel. En los primeros pacientes respetábamos la arteria espermática cuando esta era bien definida, actualmente realizamos la ligadura en bloque teniendo en cuenta que hemos tenido menos recidiva y $\sin$ atrofia testicular.

Se analizan las siguientes variables:

- Tiempo Quirúrgico.

- Estancia Hospitalaria.

- Uso de Analgésicos Post operatorios.

- Complicaciones.

- Incorporación a las actividades sociolaborales.

\section{RESULTADOS}

Total: 120 Pacientes (Tabla I).

Edad: Entre 18 y 42 años.

Tiempo quirúrgico promedio: 25.7 Minutos (Figural).

Estancia hospitalaria: (Figura 2).

Analgesia postoperatoria: Solo tres pacientes requirieron tratamiento del dolor, los cuales fueron muy

TABLA I. TOTAL DE PACIENTES Y LADO AFECTO SEGÚN DOPPLER TESTICULAR.

\begin{tabular}{|c|c|c|c|}
\hline Lado & Izquierdo & Derecho & Bilateral \\
\hline Pacientes Total: 120 & 97 & 4 & 19 \\
\hline
\end{tabular}




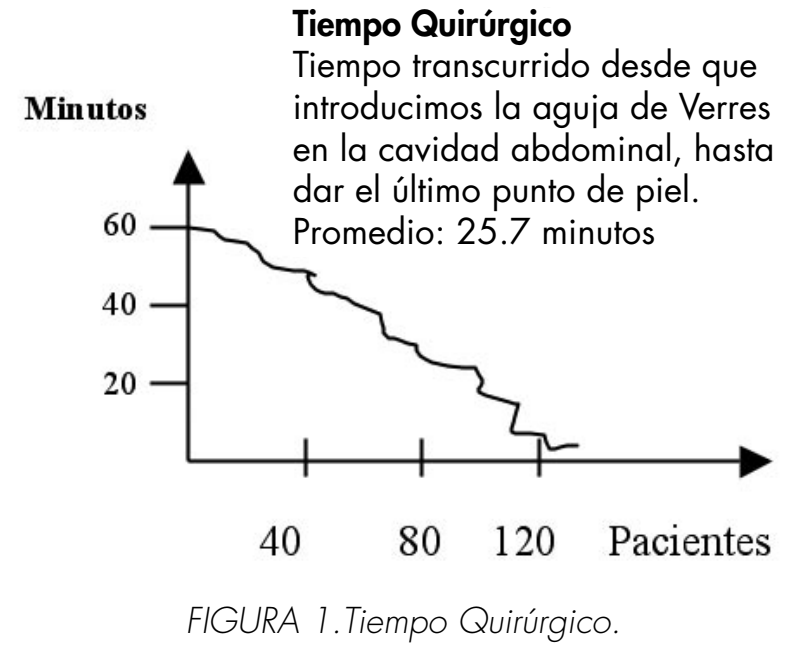

manipulados por presentar adherencias del colon sigmoide a nivel del anillo inguinal por operaciones intra-abdominales previas (Figura 3).

\section{Complicaciones}

Se presentaron 3 complicaciones menores, un enfisema subcutáneo, relacionado con insuflación del $\mathrm{CO}_{2}$ debido al paso del gas a través del anillo inguinal, que fue resuelto satisfactoriamente en el propio quirófano con variaciones de mecánica ventilatoria. Los demás pacientes sufrieron orquiepididimitis que resolvieron fácilmente con la administración de antinflamatorios. No se presentaron complicaciones mayores.

Todos los pacientes tuvieron una rápida reincorporación a las actividades sociolaborales, entre 15 y 21 días.

\section{DISCUSIÓN}

Es cierto que el tratamiento quirúrgico del varicocele por vía laparoscópica precisa de anestesia general orotraquial y la instrumentación de la cavidad peritoneal, a pesar de la experiencia del cirujano expone al paciente a posibles complicaciones (lesiones intestinales y vasculares).

Siendo eternos enamorados de las técnicas laparoscópicas en nuestra especialidad, pensamos que las indiscutibles y ya demostradas ventajas de esta técnica, no deben ser cuestionadas por excesos de entusiasmo, indicaciones incorrectas o inadecuada aplicación de esta técnica. Por lo que considero que esta técnica "no" constituye el tratamiento de elección para corregir el varicocele en el adulto, pero en indicaciones muy precisas constituye una opción terapeútica a tener en cuenta.

\section{Estancia Hospitalaria}

Horas Sólo un pequeño grupo egresaron al día siguiente por dificultades

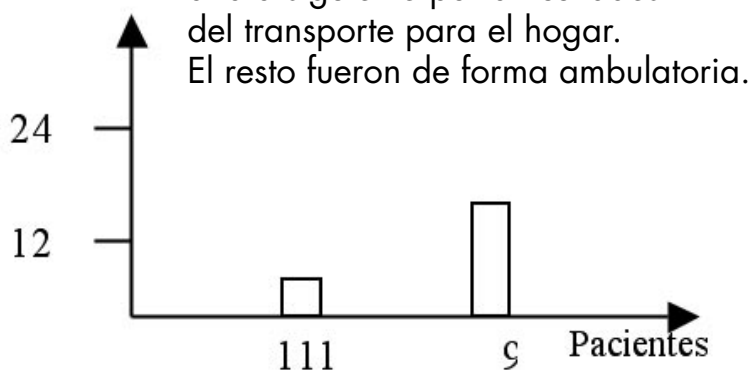

FIGURA 2. Estancia Hospitalaria.

\section{CONCLUSONES}

Consideramos que la ligadura laparoscópica de las venas espermáticas, puede considerarse como "método muy eficaz" en el tratamiento del varicocele.

\section{Ventajas}

- Magnificación del campo operatorio, permitiendo una mejor visulización de los vasos espermáticos, así como la detección de ramas venosas colaterales, disminuyendo las complicaciones testiculares y las recidivas de la enfermedad.

- Eventual uso de analgésicos.

- Estancia hospitalaria reducida.

- Rápida reincorporación a las actividades socio-laborales.

\section{Como "método de elección".}

- Varicocele bilateral.

- Pacientes obesos.

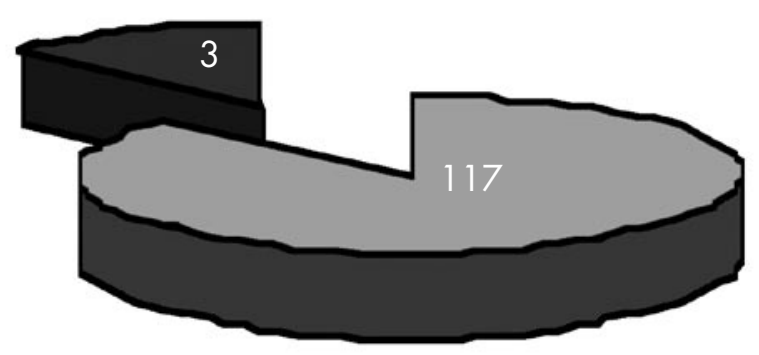

Sin Analgesia

Con Analgesia

FIGURA 3. Uso de analgésicos postoperatorio. 
- En la necesidad de tratamientos laparoscópicos simultáneos de otras patologías.

- Antecedentes de cirugía inguinal.

- Entrenamiento (Curva de aprendizaje) y

- FRACASO de las técnicas convencionales.

\section{BIBLIOGRAFÍA y LECTURAS RECOMENDADAS ( ${ }^{*}$ lectura de interés $y^{* *}$ lectura fundamental)}

**1. VALDIVIA URIA, J.G.; SÁNCHEZ SALABARDO, M.; RAMÍREZ FABIAN,M. y cols.: "Tratamiento del varicocele por laparoscopia". Arch. Esp. Urol., 57: 962, 2004.

**2. CASANOVA LÓPEZ, G.; GONZALEZ LEON, T.; DE LA CONCEPCIÓN GOMEZ, O.: "Varicocelectomía laproscópica en el adulto. Primera experiencia en Cuba". Arch. Hosp. Univ. Gral. Calixto Garcia, 2: 34, 2004.

3. IVANISSEVICH, O.: "Left varicocele due to reflux ,experience with 4470 cases y 42 years.". Int. Coll. Cir., 24: 742, 1960.

4. JIMENÉZ CRUZ, J.F.; ALLONA, A.; NAVIO, S. ycols.: "Modificación de la técnica de Buntz en la cirugía del varicocele: Resultados". Actas Urol. Esp., 7: 401, 1983.

5. PALOMO, A.: "Radical cure of varicocele: Preliminary report". J. Urol., 61: 604, 1969.

6. ALVAREZ, E.; GONZÁLEZ, P.; GARCÍA-HIDALGO, E. y cols.: "Embolización: Alternativa al tratamiento quirúrgico del varicocele." Actas Urol. Esp., 7: 481, 1983.

7. BIGOT, J.M.; JOUSSE, D.; CARETTE, M.F. y cols.: "Sclerose des varicoceles. A propors de 132 cas". Ann. Urol., 19: 393, 1985.

8. HUNTER, D.W.; CASTAÑEDA-ZÚNIIGA, W.R.; COLEMAN, C.C. y cols.: "Espermatic vein embolization with hot contrast médium or detachable ballons". Sem. Intervent. Rad., 1: 163, 1984.

9. SÁNCHEZ DE BADAJOZ, E.; DÍAZ RAMÍREZ, F.; MARÍN MARTÍN, J.: "Tratamiento endoscópico del varicocele". Arch. Esp. Urol., 41: 15, 1988.

10. WINFIELD, H.N.; DONOVAN, J.F.; SEE, W.A. y cols.: "Urological Laparoscopic Surgery". J. Urol., 146: 941, 1991.

11. WUERNSCHIMMEL, E.; LIPSKY, H.; NOEST, G.: "Laparoscopic varicocele ligation: A recomendablestandard procedure with good long-term results". Eur. Urol., 27: 18, 1995.

12. CHIU, A.W.; HUANG, W.J.; CHEN, K.K. y cols.: "Laparoscopic ligation of bilateral spermatic varices under epidural anesthesia”. Urol. Int., 57: 80, 1996.

13. DONA, B.; CANTELE, P.; PIANALTO, S. y cols.: "Laparoscopic Surgery of varicocele. Role of total endovenous anestesia in same-day discharge". G. Chir.,21: 405, 2000.

14. ZATURA, F.; STUDENT, V.; FIALA, R. y cols.: "Personal experience with peroperative study of testicular blood flow in laparoscopic surgery of varico- cele". Rozhl. Chir., 76: 419, 1977.

15. HIRSCH,I.H.;ABDEL-MEGUID,T.;GOMELLA,L. G.: "Gasless laparoscopic varicocele ligation: Experience with new instrumentation and technique forretroperitoneal and intraperitoneal approaches". J. Laparoendosc. Adv. Surg. Tech. A., 7: 221, 1997.

16. SASAGAWA, I.; YAZAWA, H.; SUZUKI, Y. y cols.: "Laparoscopic varicocelectomy in adolescents using an ultrasonically activated scalpel". Arch. Androl., 45: 91, 2000.

**17. SÁNCHEZ DE BADAJOZ, E.; JIMÉNEZ GARRIDO, A.: "Microlaparoscopic varicocelectomy". Arch. Esp. Urol., 55: 659, 2002.

*18. SÁNCHEZ DE BADAJOZ, E.; DÍAZ RAMÍREZ, F.; VARA THORBECK, C.: "Tratamiento laparoscópico del varicocele". Arch. Esp. Urol., 44: 623, 1991.

*19. SÁNCHEZ DE BADAJOZ, E.: "Cirugía laparoscópica en Urología”. Arch. Esp. Urol., 46: 541, 1993.

20. JIMÉNEZ GARRIDO, A.; GARCÍA DE LA TORRE, M.V.; SÁNCHEZ DE BADAJOZ, E.: "Una década en la varicocelectomía laparoscópica: Costes y fases de aprendizaje". Arch. Esp. Urol., 52: 245, 1999.

*21. VALDIVIA URÍA, J.G.; BAYO OCHOA, A.: "La oclusión laparoscópica de las venas espermaticas (CMI del aparato genital masculino)". Valdivia Uría JG (ed), Cirugía Mínimamente Invasiva en Urología.183. Ene Ediciones, Madrid. 1993.

22. MATSUDA, T.; YOSHIDA, O.: "Laparoscopic varicocelectomy".Arch. Esp. Urol., 46: 585, 1993.

23. LUQUE MIALDEA, R.; MARTÍN- CRESPO IZQUIERDO, R.: "Laparoscopia en urología pediátrica". Arch. Esp. Urol., 55: 737, 2002.

24. MANDRESSI, A.; BUIZZA, C.; ANTONELLI, D. y cols.: "Is laparoscopy a worthy method to treat varicocele? Comparison between 160 cases of two-portlaparoscopic and 120 cases of open inguinal spermatic vein ligation”. J. Endourol., 10: 435, 1996.

25. ISELIN, C.E.; ALMAGBALY, U.; BORST, F. y cols.: "Safety and efficiency of laparoscopic varicocelectomy in one hundred consecutive cases". Urol. Int., 58: 213, 1977.

26. ESPOSITO, C.; MONGUZZI, G.L.; GONZÁLEZSABÍN, M.A. y cols.: "Laparoscopic treatment of pediatric varicocele: A multicenter study of the Italian society of video Surgery in infancy”. J. Urol., 163: 1944.

27. PODDOUBNYI, I.V.; DRONOV, A.F.; KOVARSKII,S.K. y cols.: "Laparoscopic ligation of testicular veins for varicocele in children. Areport of 180 cases". Surg. Endosc., 14: 1107, 2000.

28. VARLET, F.; BECMEUR, F.: "Laparoscopic treatment of varicoceles in children. Multicentric prospective study of 90 cases". Eur. J. Pedriatr. Surg., 11: 399, 2001.

29. POLITO, D.S.; FLORIO, G.; CISTERNINO, S. y cols.: "Videolaparoscopic treatment of varicocele: Retrospective study of 165 cases". Chir. Ital., 53: 841, 2001. 